\title{
Sources of error in measuring cerebrospinal fluid formation by ventriculocisternal perfusion
}

\author{
A L B ER T N. MARTINS, NOR W Y N N W B Y, A N D \\ THO M A S F. D O Y L E
}

From the Neurosurgery Service, Walter Reed Army Medical Center, Washington DC, and

Neurobiology Department, Armed Forces Radiobiology Research Institute, Bethesda, Maryland, USA

SUMMARY Ventriculocisternal perfusion is regarded as a precise method of measuring the rate of formation of cerebrospinal fluid (CSF) but it possesses inherent potential sources of error. Using the technique to measure CSF formation rate in the rhesus monkey, we have observed rate changes when none were expected. Most puzzling has been the steady decline of CSF formation rate at $4 \%$ each hour during the final five hours of a seven hour perfusion although variables known to affect CSF formation remained stable. In addition, alterations in rate caused by artefacts were observed in experiments in which craniospinal blood volume was changed by sudden changes of either $\mathrm{PCO}_{2}$ or central venous pressure. Mobilisation or sequestration of incompletely equilibrated CSF is believed responsible. In other experiments, a small increase of intracranial pressure produced by increasing outflow resistance was quickly followed by an apparent reduction of CSF formation. We have concluded that to assess accurately the effect a variable has on the rate of CSF formation, one must control perfusion time and craniospinal blood volume as well as intracranial pressure.

Since the original description of the technique by Pappenheimer et al. (1962), ventriculocisternal perfusion has been considered a 'precise and physiological method for studying cerebrospinal fluid (CSF) secretion and absorption' (Lorenzo et al., 1970). Using the technique to measure rate of formation of CSF in rhesus monkeys (Martins et al., 1975), we have observed unexpected rate changes. It became apparent to us that the technique possesses inherent potential sources of error that have not been discussed adequately in the literature. This communication identifies these potential sources of error in our experimental ventriculocisternal perfusion data, and discusses their mechanisms and significance.

\section{Methods}

Rhesus monkeys (Macaca mulatta), unselected as to sex and weighing 3 to $5 \mathrm{~kg}(3.7 \pm 0.51 \mathrm{SD})$, were initially sedated with an intramuscular injec-

Address for reprint requests: Dr A. N. Martins, Neurosurgery Service, Walter Reed Army Medical Center, Washington DC 20012. USA.

Accepted 29 November 1976 tion containing phencyclidine $2 \mathrm{mg} / \mathrm{kg}$ and atropine sulphate $0.5 \mathrm{mg}$. After endotracheal intubation they were placed prone in a sphinxlike position in a stereotactic apparatus. Respiration was supported thereafter by a small animal respirator. From a catheter passed through the femoral artery into the abdominal aorta, blood pressure was monitored continuously, and arterial blood was sampled every half hour for immediate direct measurement of $\mathrm{PCO}_{2}, \mathrm{PO}_{2}$, and $\mathrm{pH}$. A sample of venous blood was taken at the beginning and end of 14 of the 18 seven hour perfusion experiments to measure serum osmolality. An externally applied heating pad kept the rectal temperature at $38^{\circ} \pm 1^{\circ} \mathrm{C}$. Lactated Ringer's solution was infused continuously by catheter into the femoral vein at $5 \mathrm{ml} / \mathrm{kg} / \mathrm{h}$. In one group of animals, anaesthesia was maintained with small doses of pentobarbitone given intravenously as required. In a second group, anaesthesia was maintained with an inspired gas mixture of $75 \%$ nitrous oxide and $25 \%$ oxygen, supplemented by a continuous intravenous infusion of tubocurarine chloride $0.4 \mathrm{mg} / \mathrm{kg} / \mathrm{h}$. In some animals of this 
group $\mathrm{PCO}_{2}$ was decreased by hyperventilation or increased by adding $\mathrm{CO}_{2}$ to the inspired gas mixture.

A 22-gauge needle was passed stereotactically into the left lateral ventricle and a 19-gauge needle, connected to a polyethylene tube, was passed percutaneously into the cisterna magna. The outflow end of the polyethylene tube was positioned at 1 to $5 \mathrm{~cm}$ below the cisterna magna. The perfusion fluid was a balanced salt solution (Martins et al., 1975) with $\mathrm{pH}$ adjusted to 7.35 before use by exposure to $5 \% \mathrm{CO}_{2}$ in oxygen. To each $\mathrm{ml}$ of perfusion fluid was added $1 \mathrm{mg}$ of blue dextran (molecular weight $=2$ million). The perfusion fluid, warmed to $38^{\circ} \mathrm{C}$, was infused by pump through the ventricular needle at a rate calibrated gravimetrically to an accuracy of $0.0001 \mathrm{ml} / \mathrm{min}$. Throughout each experiment the perfusion rate varied by less than $0.001 \mathrm{ml} / \mathrm{min}$. For those animals anaesthetised with pentobarbitone, the mean perfusion rate was $0.18 \mathrm{ml} / \mathrm{min}$, and for those anaesthetised with nitrous oxide, the perfusion rate was $0.23 \mathrm{ml} / \mathrm{min}$. A pressure transducer connected in parallel to the ventricular inflow tube continuously monitored the intraventricular CSF pressure, which never rose above $7 \mathrm{mmHg}$ and usually varied by less than $3 \mathrm{mmHg}$ for each experiment. Effluent flowing from the needle in the cisterna magna was discarded during the first two hours of each perfusion experiment. For the remainder of each experiment, the effluent was collected in paraffin-covered tared vials as separate specimens each $0.5 \mathrm{~h}$. Each specimen was weighed to an accuracy of $0.1 \mathrm{mg}$ to determine the outflow rate, and then centrifuged at $3600 \mathrm{rpm}$ for $6 \mathrm{~min}$ to remove cellular debris. The relative concentration of blue dextran in both supernate and perfusion fluid was determined by measuring their optical densities with spectrophotometer (Beckman, DB) at a wavelength of $610 \mathrm{~nm}$. The average rate of formation of CSF $\left(V_{t}\right)$ for each half hour period was calculated using the formula derived by Heisey et al. (1962):

$$
\mathrm{V}_{\mathbf{i}}=\mathrm{Vi} \frac{\mathrm{Ci}-\mathrm{Co}}{\mathrm{Co}}
$$

in which $\mathrm{Ci}$ and $\mathrm{Co}$ are the concentrations of blue dextran in the inflow and outflow fluid respectively, and $\mathrm{Vi}$ is the perfusion rate.

At the end of each experiment the animal was killed with an overdose of pentobarbitone, and its brain was removed to verify that valid ventriculocisternal perfusion had been accomplished.

In most experiments, a seven hour perfusion was completed in which blood pressure, intracranial pressure, temperature, and blood gases were kept stable and within normal limits. In a few additional experiments, the intracranial pressure was transiently increased deliberately, either by increasing central venous pressure with a rapid infusion of homologous whole blood into the inferior vena cava, or by raising the height of the outflow tubing. In other experiments, $\mathrm{PCO}_{2}$ was abruptly increased by adding $\mathrm{CO}_{2}$ to the inspired gas mixture or reduced by hyperventilation.

\section{Results}

In 18 monkeys a satisfactory seven hour ventriculocisternal perfusion was completed in which temperature, blood gases, blood pressure, and intracranial pressure did not change significantly and remained within normal limits. Mean serum osmolality at the start of the perfusion was 302 milliosmols ( \pm 5.3 SD) and at the end it was 303 milliosmols ( $\pm 8.7 \mathrm{SD})$. This difference is not statistically significant $(\mathrm{P}=>0.5$, paired $t$ test; $\mathrm{n}=14$ ). Five of the 18 monkeys were anaesthetised with nitrous oxide and perfused at $0.23 \mathrm{ml} / \mathrm{min}$, and the remainder were anaesthetised with pentobarbitone and perfused at $0.18 \mathrm{ml} / \mathrm{min}$. There were no differences in the CSF formation rates between these two groups so the data were combined and analysed together. The Table lists the average CSF formation rates of these 18 monkeys during each $0.5 \mathrm{~h}$ of the last five hours of perfusion. Linear regression analysis (method of least squares) of mean CSF formation rates as a function of duration of perfusion yields a line described by the equation:

$$
y=-1.7 x+39.2
$$

in which $x=$ time in hours with the end of 2.5 hours of perfusion taken as zero. The slope of this line is significantly different from zero $(P<0.001$; Fig. 1). When intracranial pressure was increased briefly by increasing central venous pressure, outflow rate increased transiently, and

Table CSF formation rate at half hour intervals during prolonged ventriculocisternal perfusion

\begin{tabular}{ll}
\hline Collection period $\dagger$ & Rate $\mu \mathrm{l} / \mathrm{min}^{*}$ \\
\hline $2-2.5$ & $39.2 \pm 6.00$ \\
$2.5-3$ & $38.8 \pm 5.07$ \\
$3-3.5$ & $36.9 \pm 5.02$ \\
$3.5-4$ & $37.3 \pm 8.05$ \\
44.5 & $35.7 \pm 5.23$ \\
$4.5-5$ & $35.2 \pm 6.57$ \\
$5-5.5$ & $33.5 \pm 4.63$ \\
$5.5-6$ & $33.6 \pm 4.02$ \\
$6-6.5$ & $32.9 \pm 4.15$ \\
$6.5-7$ & $31.8 \pm 3.83$ \\
\hline
\end{tabular}

- Mean \pm standard deviation of 18 experiments.

$\dagger$ In hours from beginning of perfusion. 


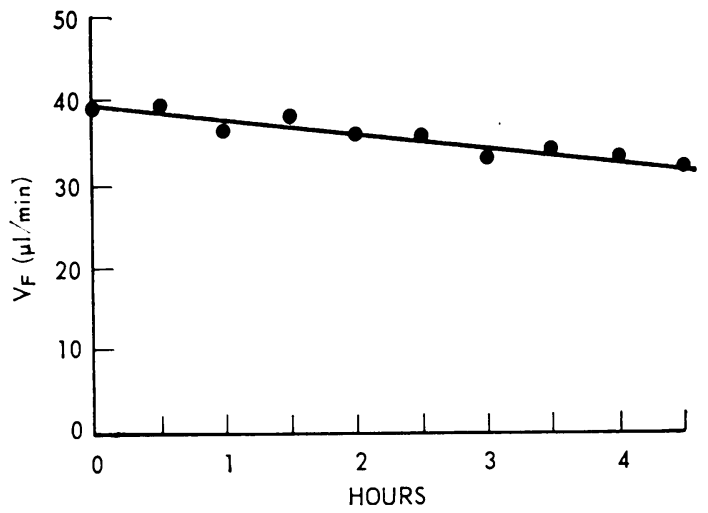

Fig. 1 Linear regression analysis (least squares method) of mean CSF formation rate $\left(V_{1}\right)$ plotted against duration of perfusion. $Y=-1.7 x+39.2$, in which $x=$ time in hours with the end of 2.5 hours of perfusion taken as zero. Slope significantly different from zero $(\mathrm{P}<0.001)$.

concurrently the CSF formation rate, calculated from indicator dilution, also increased (Fig. 2). After step increase of $\mathrm{PCO}_{2}, \mathrm{CSF}$ formation rate transiently increased, and after step decrease of $\mathrm{PCO}_{2}$, the rate transiently decreased (Fig. 3). When the height of the outflow tubing was raised and then lowered, intracranial pressure increased and then decreased. This change in intracranial pressure was followed by a reciprocal change in the apparent CSF formation rate (Fig. 4).

\section{Discussion}

The rate of formation of CSF was found to decline steadily at about $4 \%$ each hour during the last five hours of a seven hour ventriculocisternal perfusion although physiological parameters thought to influence the rate, such as intracranial pressure (Hochwald and Sahar, 1971), blood pressure (Carey and Vela, 1974), temperature (Snodgrass and Lorenzo, 1972), and $\mathrm{PCO}_{2}$ (Ames et al., 1965; Oppelt et al., 1963) were kept stable. An adequate explanation for this unexpected finding has eluded us. We speculated that perhaps pentobarbitone anaesthesia was responsible for progressively depressing the rate. This explanation was rejected because CSF formation rate declined at a similar rate in animals anaesthetised with nitrous oxide. The declining rate was also suspected of being an artefact of delayed equilibration of perfusion fluid with the original CSF pool. This is unlikely in view of the relatively rapid perfusion rate employed compared to the small total volume of CSF in the monkey. Moreover,
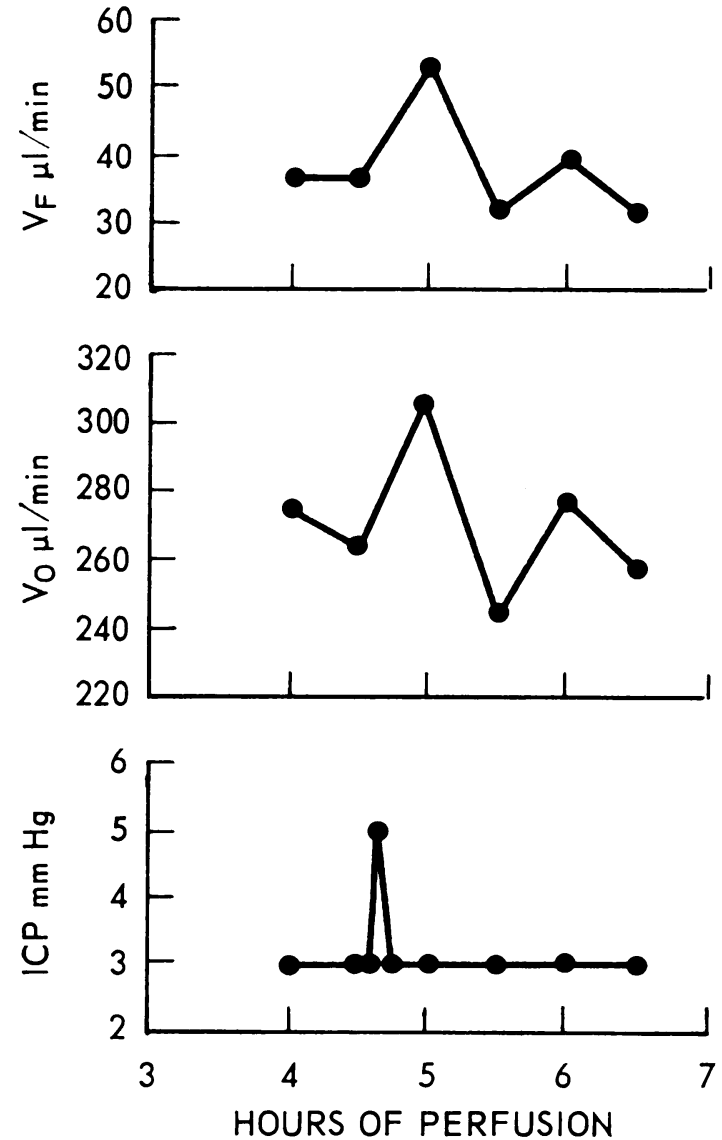

Fig. 2 Brief rise in intracranial pressure (ICP), induced at 4.6 hours of perfusion by increasing central venous pressure with a rapid infusion of blood into the inferior vena cava, is accompanied by $a$ transient apparent increase in CSF formation rate $\left(V_{\mathrm{f}}\right)$ and outflow volume $(V o)$ presumably caused by mobilisation of CSF from the spinal sac (perfusion rate $=0.237 \mathrm{ml} / \mathrm{min}$ ).

increasing the perfusion rate by $30 \%$ to reduce equilibration time had no effect. CSF formation rate declined at about the same rate whether the perfusion rate was $0.18 \mathrm{ml} / \mathrm{min}$ or $0.23 \mathrm{ml} / \mathrm{min}$. Finally, progressive dehydration was considered as a possible cause but had to be rejected in view of the stable serum osmolality throughout the experiment. In any event, whether the steadily declining rate is real or only an artefact of our experimental method, our observations suggest that unless suitable controls show otherwise, time should be considered as another variable that may influence CSF formation rate during prolonged ventriculocisternal perfusion. 

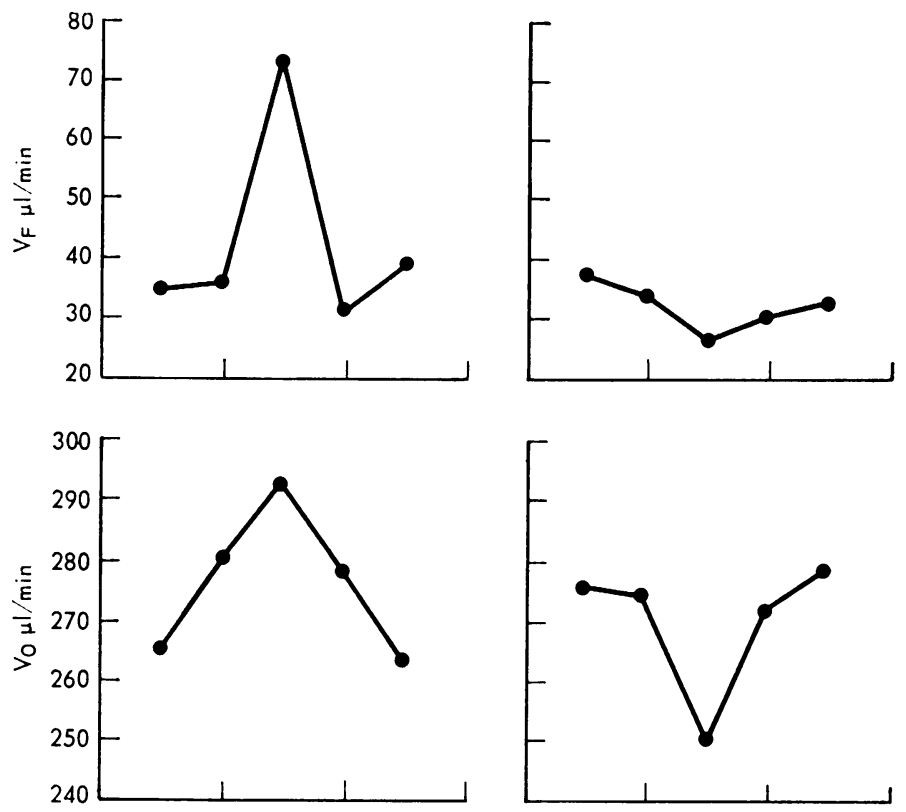

Fig. 3 Left: step hypercapnia at the fifth hour of perfusion is accompanied by a transient apparent increase in both CSF formation rate and outflow volume (perfusion rate $=0.239 \mathrm{ml} / \mathrm{min}$ ). Right: the reverse is true for step hypocapnia at the third hour of perfusion (perfusion rate $=0.237 \mathrm{ml} / \mathrm{min}$ ).

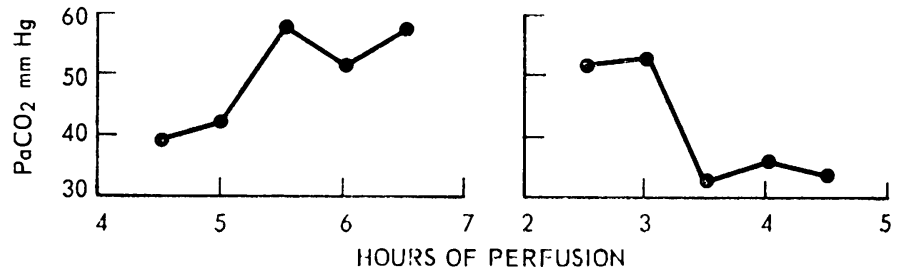

The rate of CSF formation fluctuated widely from sample to sample in response to changes in central venous pressure, to changes in cerebral blood volume induced by step hypocapnia or hypercapnia, and to small changes in outflow resistance that changed intracranial pressure (Figs. 2-4). These fluctuations took place long after the concentration of the blue dextran indicator in the outflow fluid was essentially stable. If correlated with the anatomy and physiology of the CSF-containing space, these observations can be readily explained as follows: CSF is contained within a multi-chambered cavity - that is, ventricles and the craniospinal subarachnoid spacewhich hinders rapid and complete mixing of perfusion fluid with the entire reservoir of CSF. Even after seven hours of perfusion from lateral ventricle to cisterna magna, pools of unequilibrated CSF probably remain isolated in sulci, cisterns, the spinal sac, and other culs-de-sac outside the main perfusion stream. These sequestered pools may be mobilised to effect spatial compensation when craniospinal blood volume changes. Normally, CSF moves to and fro between the cranium and the spine in response to changes in craniospinal blood volume, the spinal sac acting as a bladder-like reservoir of variable capacity (Martins et al., 1972). However, during ventriculocisternal perfusion, CSF that is normally dislocated cephalad or caudad will tend to flow out of the open needle in the cisterna magna. If dislocated CSF had not equilibrated with the perfusion fluid, it would dilute blue dextran in the outflow fluid. This can explain why we observe a transient increase in CSF formation rate caused by an artefact when step hypercapnia increases the cerebral blood volume (Grubb et al., 1974). Similarly, in response to a transient increase in central venous pressure, which presumably increases the volume of blood present within the spinal epidural plexus of veins, the spinal sac partially collapses (Martins et al., 1972), forcing unequilibrated CSF out through the needle in the cisterna magna. To explain the transient fall in 

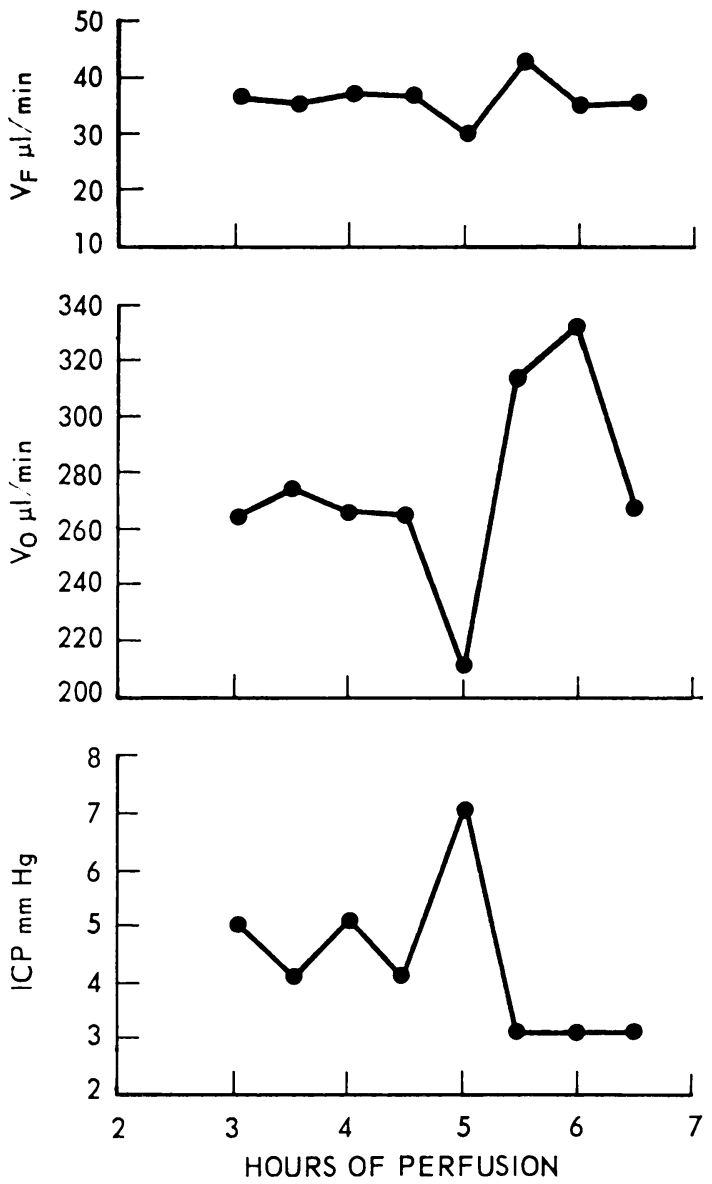

Fig. 4 Intracranial pressure is increased at the end of 4.5 hours of perfusion by increasing outflow resistance, and both CSF formation rate and outflow volume fall. When outflow resistance is decreased, intracranial pressure falls while both CSF formation rate and outflow volume transiently increase (perfusion rate $=0.232 \mathrm{ml} / \mathrm{min}$ ).

formation rate with step hypocapnia, we assume that some of the newly-formed CSF does not mix with the perfusion fluid because it is sequestrated in spaces that enlarge to compensate for the reduction in cerebral blood volume that attends hypocapnia (Grubb et al., 1974).

Changes in CSF formation rate caused by artefacts, as measured by ventriculocisternal perfusion, may also accompany alteration of intracranial pressure induced by lowering or raising outflow resistance. A sudden fall in intracranial pressure produced by lowering outflow resistance will tend to draw fluid from pools of unequilibrated CSF. The mechanism by which modest increases in intracranial pressure appear to reduce CSF formation rate is less clear. Hochwald and Sahar (1971) observed that as intracranial pressure rises in the cat, CSF formation rate decreases. They suggest that the rate falls as intracranial pressure rises because blood flow to the CSFforming areas of the brain also falls. On the other hand, the reduction in rate may be an artefact of the method. For ventriculocisternal perfusion to be a valid measure of CSF formation rate, all newly-formed CSF must be mixed with the perfusion fluid. The possibility exists that as intracranial pressure is increased, some newlyformed CSF is diverted to channels of drainage before it can thoroughly mix with perfusion fluid. This is especially likely if, as the work of Sato and Bering (1967) indicates, CSF is formed in the cranial subarachnoid spaces.

In summary, CSF formation rate in the monkey as measured by prolonged ventriculocisternal perfusion, declines spontaneously with time for reasons that remain obscure. By mobilising unequilibrated pools of CSF, sudden changes of craniospinal blood volume can lead to changes in CSF formation rate caused by artefact. And there is reason to believe that part of the alteration in rate observed after intracranial pressure is increased or decreased may also be an artefact of the method. These sources of error must be avoided or controlled if one is to assume correctly that a variable under study is responsible for an observed alteration in CSF formation rate.

Mr Sheldon Levin and Mr William Jackson performed the statistical analysis of the data. This study was supported by a grant from the US Army Medical Research and Development Command.

\section{References}

Ames, A. K., Higashi, K., and Nesbitt, F. B. (1965). The effects of $\mathrm{PCO}_{2}$ acetazolamide and ouabain on volume and composition of choroid-plexus fluid. Journal of Physiology, 181, 516-524.

Carey. M. E., and Vela, A. R. (1974). The effect of systemic arterial hypertension on the rate of cerebrospinal fluid formation in dogs. Journal of Neurosurgery, 41, 350-355.

Grubb, R. L., Raichle, M. E.. Eichling, J. O.. and TerPogossian, M. M. (1974). The effects of changes in $\mathrm{PaCO}_{2}$ on cerebral blood volume, blood flow, and vascular mean transit time. Stroke, 5, 630-639.

Heisey. S. B., Held, D., and Pappenheimer, J. R. (1962). Bulk flow and diffusion in the cerebrospinal fluid system of the goat. American Journal of Physiology, 203, 775-781.

Hochwald, G. M., and Sahar, A. (1971). Effect of 
spinal fluid pressure on cerebrospinal fluid formation. Experimental Neurology, 32, 30-40.

Lorenzo, A. V., Page, L. K., and Watters, G. V. (1970). Relationship between cerebrospinal fluid formation, absorption and pressure in human hydrocephalus. Brain, 93, 679-692.

Martins, A. N., Ramirez, A., and Doyle, T. F. (1975). Comparison of radio-iodinated serum albumin and blue dextran as indicators to measure rate of formation of cerebrospinal fluid. Experimental Neurology, 47, 249-256.

Martins, A. N., Wiley, J. K., and Myers, P. W. (1972). Dynamics of the cerebrospinal fluid and the spinal dura mater. Journal of Neurology, Neurosurgery, and Psychiatry, 35, 468-473.
Oppelt, W. W., Maren, T. H., Owens, E. S., and Rall D. P. (1963). Effects of acid-base alterations on cerebrospinal fluid production. Proceedings of the Society of Experimental Biology and Medicine, 114, 86-89.

Pappenheimer, J. R., Heisey, S. R., Jordan, E. F., and Downer, J. deC. (1962). Perfusion of the cerebral ventricular system in unanesthetized goats. American Journal of Physiology, 203, 763-774.

Sato, O., and Bering, E. A. (1967). Extra-ventricular formation of cerebrospinal fluid. Brain and Nerve (Tokyo), 19, 883-885.

Snodgrass, S. R., and Lorenzo, A. V. (1972). Temperature and cerebrospinal fluid production rate. American Journal of Physiology, 222, 1524-1527. 